\title{
LOS CONGRESOS MEXICANOS DE BOTANICA DE 1960 A 1978
}

\section{SERGIO GUEVARA*}

\section{PATRICIA MORENO-CASASOLA}

El Congreso Mexicano de Botánica, organizado por la Sociedad Botánica de México desde octubre de 1960 , se ha realizado cada 3 años, habiéndose efectuado el último en la ciudad de México en octubre de 1978. (Fig. 1).

El congreso se realiza por primera vez dada la gran actividad y entusiasmo que en el campo de la botánica se despliega en instituciones gubernamentales y de educación superior, tanto de la ciudad de México como de provincia. Esto creó la necesidad de establecer comunicación entre sí, rompiendo un estancamiento $\mathrm{y}$ aislamiento de algunios años y aprovechando la gran capacidad organizativa de algunos botánicos, entre los que destaca el Dr. Jerzy Rzedowski. Este acontecimiento obedece a varios factores como son la labor desarrollada por distinguidos botánicos como el Ing. Efraím. Hernández Xolocotzi y el Dr. Faustino Miranda; este último impulsó, por primera vez en México, la formación sistemática de profesionales de la botánica a nivel de grado y de postgrado, relacionando estrechamente centros de investigación (Instituto de Biología, UNAM; Instituto Nacional de Investigaciones Forestales, SAG) con un centro de docencia (Facultad de Ciencias, UNAM). Procuró la participación de los botánicos en la docencia incorporando egresados de la Facultad a los programas de investgiación, iniciándose así la formación de grupos de investigación en México. Incidió asimismo, en la formación de profesionales trasmitiendo de manera entusiasta, clara y precisa los conceptos básicos de la biología, demostrando una metodología depurada en el planteamiento y desarrollo de la investigación, manteniendo ritmos de trabajo poco usuales y generando una gran cantidad de ideas relacionadas con las investigacionoes biológicas de mayor importancia (Hernández, 1969a). Logró cambiar el concepto básico de la orientación de la nivestigación y ampliar los campos de estudio de la botánica (Hernández, 1969a), ligando la investigación básica con programas gubernamentales (Gómez Pompa, 1975), demostrando asimismo, fundamentalmente a través de estudios florísticos de vegetación y taxonómicos, la necesidad de incorporar los grupos de investigación botánica al inventario de los recursos naturales del país. De esta manera logró que la botánica ocupara el lugar que le corresponde en el impulso científico que mueve a México (Miranda, 1961). Al mismo tiempo pugnó por la formación de grupos interdisciplinarios, en sus palabras “...impulsar los trabajos en conjunto en una determinada región donde se aborden de manera armónica los diversos problemas naturales de la misma y sus relaciones con las actividades humanas..." (Miranda, 1961). Esta actitud fortaleció la participación de otros institutos y centros de investigación los cuales funcionaron como auxiliares del trabajo botánico (Hernández, 1969a).

- Laboratorio de Ecología. Facultad de Ciencias, UNAM. 
Fig. 1. Datos generales de los Congresos Mexicanos de Botánica.

CONGRESO

I
II
III
IV
${ }^{*}$ V
VI
VII

FECHA

24-26 Oct. 1960

17-21 Sept. 1963

24-28 Oct. 1966

8-11 Sept. 1969

3-9 Dic. 1972

21-26 Sept. 1975

15-21 Oct. 1978
SEDE

Cd. de México

San Luis Potosí

Cd. de México

Monterrey-Saltillo

Cd. de México

Jalapa

Cd. de México
No. de Trabajos

74

97

75

102

207

172

183

- Se organizó conjuntamente con el I Congreso Lationamericano de Botánica.

Se tomaron en cuenta presentaciones libres y symposia tanto nacionales como extranjeros. En varios casos el número total obtenido no coincide con la numeración final de los trabajos en los resúmenes debido a errores en la secuencia. 
Fig. 2. Número de trabajos presentados por área en cada Congreso, excluyendo los trabajos presentados por personas o instituciones extranjeras.

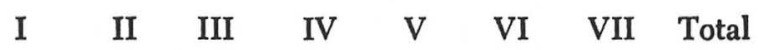

$\begin{array}{lrrrrrrrr}\text { Taxonomía y Florística } & - & 6 & - & - & 13 & 25 & 31 & 75 \\ \text { Botánica Criptogámica } & 4 & 5 & 5 & 12 & - & - & - & 26 \\ \text { Botánica Fanerogámica } & 7 & 4 & 5 & 16 & - & - & - & 32 \\ \text { Ecología } & - & 13 & 20 & - & 23 & 52 & 61 & 169\end{array}$

Fitogeografía y Florística $-\quad-\quad 6 \quad-\quad-\quad-\quad-6$

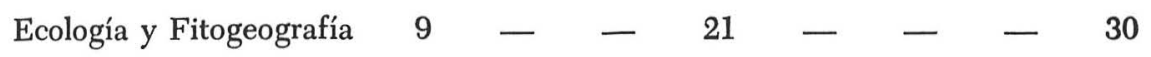

Fitogeografía $\quad-\quad 7 \quad-\quad-\quad 3 \quad-10$

Historia, Bibliografía y

Colecciones

Morfología y Anatomía

Fisiología

$\begin{array}{llllllll}- & 2 & 6 & 3 & 2 & 4 & 7 & 24\end{array}$

Fitoquímcia

Paleobotánica

$\begin{array}{llllllll}1 & 2 & 5 & 3 & 13 & 21 & 14 & 59\end{array}$

$\begin{array}{llllllllll}\text { Enseñanza de la Botánica } & 2 & - & - & - & 2 & 6 & 5 & 15\end{array}$

Citogenética

$\begin{array}{llll}5 & 4 & 7 & 3\end{array}$

Etnobotánica

Botánica Económica

53

$\begin{array}{lllllll}2 & 7 & 9 & 15 & 11 & 52\end{array}$

Fitopatología y Micología

$$
\text { T o t a } 1
$$

49

-
5
2
5

8

$3 \quad 11$

$\begin{array}{llllllll}- & - & - & - & 2 & 17 & 27 & 46\end{array}$

$\begin{array}{llllllll}11 & 19 & 9 & 11 & 6 & - & -\quad 56\end{array}$

$\begin{array}{lllll}6 & 2 & - & 4\end{array}$ 
La relación establecida con organismos gubernamentales generó una cierta cantidad de recursos que fueron utilizados para incorporar nuevo personal, así como para impulsar su formación a nivel de postgrado en el extranjero y como ya se mencionó antes, para crear cierta infraestructura como por ejemplo: apoyo al Herbario Nacional, incorporación de computadoras para análisis y acumulación de información, creación de jardines botánicos, invernaderos y reservas biológicas lo cual permitía al mismo tiempo realizar investigación en condiciones controladas, montar experimentos en lugares fijos, etc. Esto se logro también gracias al apoyo decidido que le otorgaran las autoridades universitarias en aquel tiempo.

En cuanto a la biología agrícola, es importante mencionar la creación del Colegio de Postgraduados de Chapingo, producto entre otros del trabajo del Ing. Efraim Hernández Xolocotzi quien impulsó la idea de transformar la Escuela Nacional de Agricultura de Chapingo, de un centro de experimentación en un centro de investigación (Miranda, 1961). "Es conveniente dirigir esta investigación hacia logros de incremento sostenido de la producción agropecuaria y forestal. Conviene también establecer las bases para investigaciones básicas que redunden en conocimientos que sirvan para encontrar soluciones a problemas específicos de nuestro panorama agrícola sin la necesidad de depender totalmente de las corrientes de investigación de otros países" (Hernández, 1969b).

Desde el momento mismo de su realización este evento se convirtió en el foro nacional más importante para dar a conocer el producto del trabajo botánico realizado en el país por instituciones, grupos o investigadores, como se puede corroborar tanto por los temas incluidos en cada uno de los congresos, como por los 723 trabajos presentados por 5.94 autores en presentaciones personales, simposios, conferencias y mesas redondas, representando a 54 instituciones (Fig. 2 y 3). Aún cabría designar al congreso como uno de los eventos más importantes que realiza la comunidad científica del país en el área de ciencias naturales, tanto por su participación como por su antiguedad (21 años).

Este congreso ha incluido distintas disciplinas de la botánica las cuales lo han mantenido.como tribuna importante con excepción tal vez del área de Botánica Económica (agrícola y forestal) y Fisiología Vegetal; de la primera surgió el área de la Etnobotánica y se ha conservado y fortalecido debido probablemente al establecimiento de grupos de trabajo en los centros de docencia e investigación de universidades e institutos con participación de investigadores que surgen y/o se ligan al área de ecología y taxonomía -florística. Volveremos posteriormente a este punto cuando analicemos la evolución de los congresos.

Hasta este momento, la organización del Congreso Mexicano de Botánica y la publicación del Boletín se han convertido en las actividades principales de la Sociedad Botánica de México. En el presente trabajo haremos algunas reflexiones sobre 21 años de botánica analizando el contenido de los congresos, considerando los trabajos presentados, su distribución por temas y las instituciones representadas.

El análisis de la investigación botánica en México se inicia con los trabajos de Hernández (1961) y Miranda (1961) que publicaran con motivo del 25 aniversario de la Sociedarl Mexicana de Historia Natural, donde analizaron la historia y desarrollo de la biología agrícola y botánica respectivamente en esos años. E] Ing. Hernánde\% describe el estado actual y surgimiento de la investgiación 
LOS CONGRESOS MEXICANOS DE BOTANICA DE 1960 A 1978

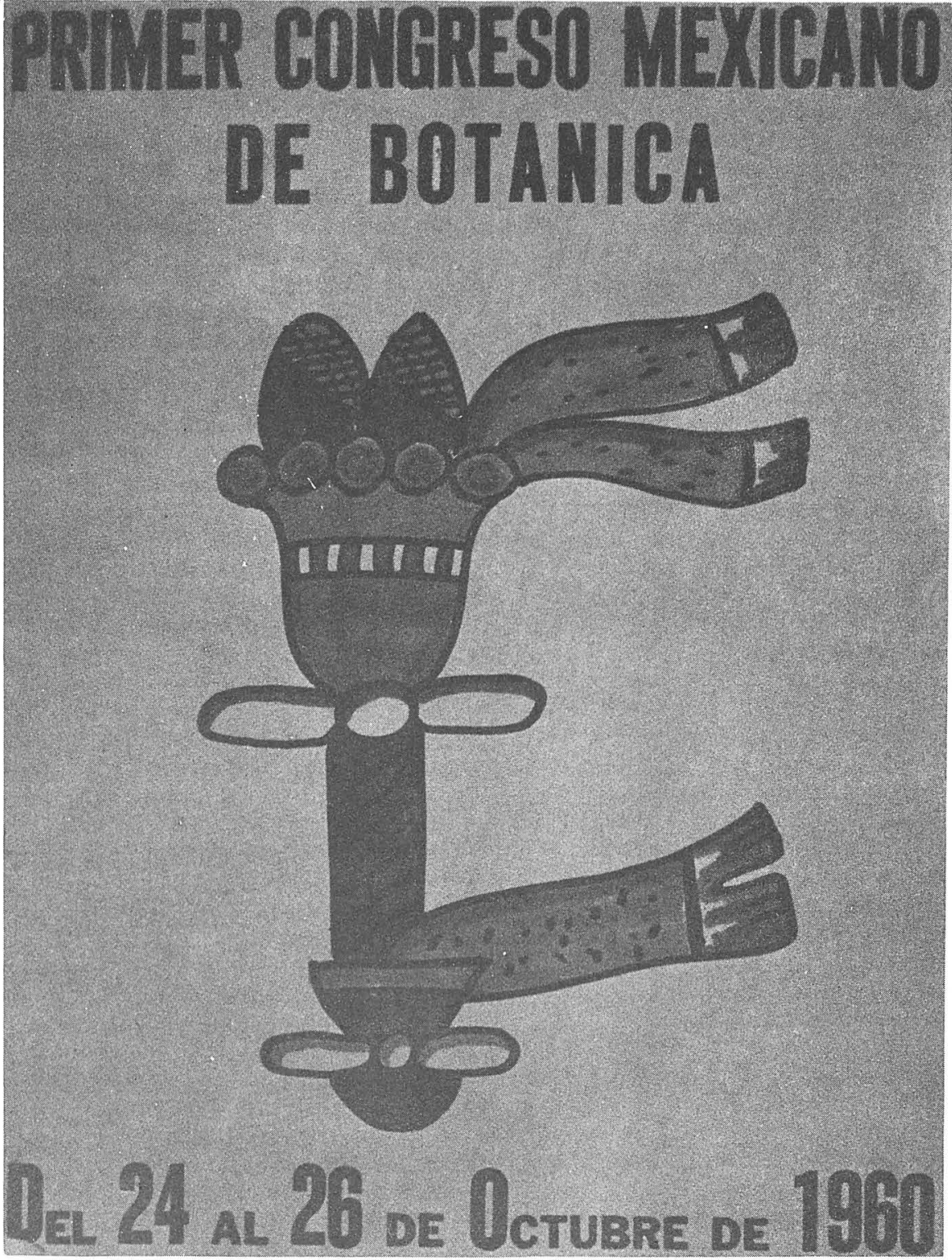

Cartel del Primer Congreso 
agrícola en el país. El Dr. Miranda describe detalladamente la producción botánica haciendo anotaciones y recomendaciones para su desarrollo fututro.

Previo a la realización del IV Congreso el Dr. James R. Wells, botánico del Instituto de Ciencias de Granbrook de Bloomfield Hills, Michigan, sugiere la idea de realizar un simposio sobre la investigación botánica en México el cual fue organizado por el Dr. Jerzy Rzedowski, (Simposio sobre la Investigación Botánica en México, IV Congreso Mexicano de Botánica), con los siguientes objetivos:

1. Informar formalmente a la comunidad botánica acerca de los proyectos botánicos de mayor alcance que se llevan a cabo en México.

2. Contribuir a la definición de necesidades de futuros proyectos.

3. Ayudar a difundir la necesidad de cooperación entre investigadores presentes y futuros que colectivamente contribuyen en la preparación de la flora de México.

En este simposio participaron el Ing. Efraim Hernández X. y el Dr. Gastén Guzmán, quienes hicieron un análiiss de las causas del desarrollo de algunas instituciones importantes, así como un inventario de los programas de investigación y recursos materiales y humanos disponibles en el Instituto de Biología (UNAM), Escuela Nacional de Agricultura y Colegio de Postgraduados de Chapingo, Instituto Nacional de Investigaciones Forestales (SAG), Instituto Nacional de Antropología e Historia, Instituto Politécnico Nacional y algunas instituciones de provincia como: Instituto de Investigaciones de Zonas Desérticas (UASLP), Instituto de Botánica (Universidad de Guadalajara), Instituto Tecnológico y de Estudios Superiores, Monterrey, N.L., y Universidad de Nuevo León, Monterrey, N.L.

Posteriormente, durante el VI Congreso el Dr. Jerzy Rzedowski dictó una conferencia intitulada "Ensayo de Evaluación de la Investigación Botánica en México", donde analiza las condiciones en las que se realiza la investigación, así como el desarrollo de distintas disciplinas y de recomendaciones para su superación. Gómez-Pompa (1975) publica un trabajo acerca de la investigación botánica en México en los últimos diez años, en el cual analiza concretamente el impacto del trabajo del Dr. Faustino Miranda en la botánica mexicana.

Este tema no sólo ha sido tratado en los congresos, sino que se ha planteado regularmente como inquietud de la comunidad botánica en simposios, conferencias y mesas redondas, y aún así no se ha generado una discusión amplia ni la elaboración de recomendaciones o lineamientos, ni la formación de grupos de trabajo o de análisis. Esta ha sido una falla lamentable que debemos subsanar.

El análisis de los congresos se inscribe en el estudio de la investigación botánica en México aunque por supuesto, es complementaria de la información acerca de las publicaciones aparecidas en revistas nacionales e internacionales y del inventario de los programas o proyectos de investgiación en curso. De todas formas presumimos que en los congresos se presenta una muestra significativa de la producción botánica; además deja ver el papel que la Sociedad Botánica de México ha tenido en la organización de los mismos y su impacto en la ciencia del país. Debemos mencionar que nuestras observaciones están limitadas por el tipo de organización de (adla (congreso. Esto es, la participación de mayor número de ponentes representando mayor número de grupos de investigación e institucio- 
LOS CONGRESOS MEXICANOS DE BOTANICA DE 1960 A 1978

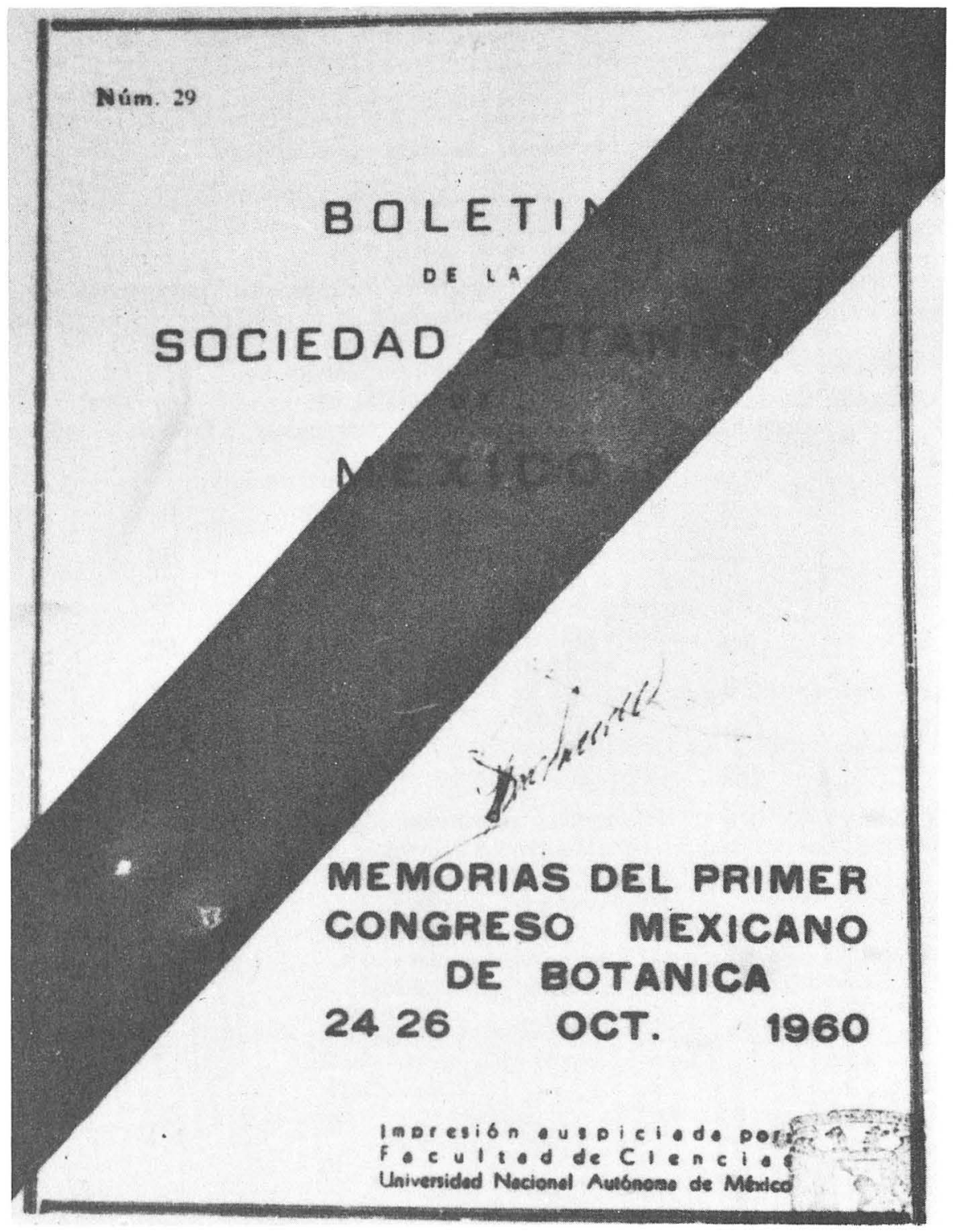

Portada de las Memorias del Primer Congreso 
BOLETIN DE LA SOCIEDAD BOTANICA DE MEXICO No. 40, 1981

Fig. 3. Número de trabajos (nacionales y extranjeros) presentados en cada congreso.

Presentaciones libres Simposios, Mesas

Congreso

Redondas, etc.

Total

nacionales extranjeros nacionales extranjeros Present. Simp.

$\begin{array}{rrrrrrr}\text { I } & 49 & 17 & 6 & 2 & 66 & 8 \\ \text { II } & 68 & 17 & 12 & - & 85 & 12 \\ \text { III } & 67 & 8 & - & - & 75 & - \\ \text { IV } & 82 & 14 & 4 & 2 & 96 & 6 \\ \text { V } & 83 & 71 & 15 & 38 & 154 & 53 \\ \text { VI } & 156 & 16 & - & - & 172 & - \\ \text { VII } & 165 & 2 & 16 & - & 167 & 16 \\ \text { T O T A L } & 670 & 145 & 53 & 42 & 815 & 95\end{array}$


Fig. 4. Simposios, conferencias y mesas redondas efectuadas durante los congresos.

Congreso:

I Simposio: Vegetación en México

II Simposio:Zonas áridas de México

Conferencias: Importancia de la botánica en la investgiación agrícola en México

La botánica y su importancia en la industria agrícola. Desarrollo y significación de la fitogenética en México

Importancia de la botánica en las investigaciones forestales

III

IV

V

Simposio: La investigación botánica en México

Simposio: Avances en los estudios paleobotánicos

Avances en los estudios florísticos

Avances en sistemática y evolución

Avances en fisiología y autoecología

Avances en estudios sinecológicos

Enseñanza de la botánica

Aportaciones de la botánica a programas de desarrollo.

Conferencias: La flora de México

Objetivos y funcionamiento de la Organización Pro Flora Neotrópica (O.F.N.)

VI Conferencias: Enseñanza de la botánica

Ensayo de evaluación de la investigación botánica en México

Utilización conservacionista de los recursos naturales

Los recursos naturales y la protección de las cuencas hidrográficas en zonas tropicales

VII Simposio: Biología de la polinización

Dinámica de poblaciones

Etnobotánica

Mesa redonda: Nomenclatura y caracterización de los tipos de vegetación 
BOLETIN DE LA SOCIEDAD BOTANICA DE MEXICO No. 40, 1981 nes depende de la promoción y previsión de las actividades programadas principalmente y de manera secundaria de la ubicación y facilidades de acceso a la sede del evento. Existe otra limitación que es el temario o apartados donde se agrupan los trabajos lo cual depende de la decisión del comité organizador correspondiente; esto nos llevó a conocer el contenido de cada trabajo con el fin de reagruparlo en categorías comunes para poder extraer ciertas conclusiones.

Cada congreso consta de dos partes básicas con sus variantes: la primera la conforman las presentaciones libres y se agrupan según un temario (en esta parte se puede analizar el trabajo de gran cantidad de personas, grupos e instituciones) y la segunda parte donde quedan comprendidos los simposios, mesas redondas y conferencias, en donde se incluyen análisis y discusiones del conocimiento botánico así como diversos aspectos de la situación actual de la botánica en el país (Fig. 4). Por lo tanto las discutiremos por separado.

A lo largo de los congresos se puede observar la aparición, fortalecimiento y desaparición de cada tema o sesión especial. Para la discusión agrupamos varios de estos temas en conjuntos que nos permitan ligar su origen y desarrollo a los grupos o instituciones que lo realizan.

Grupo Ecología-Taxonomía-Florística: Como se ha mencionado antes, la botánica a fines de la década de 1950 y principio de 1960 presenta un vigoroso desarrollo, cuenta con grupos de investigación en varias instituciones (UNAM, IPN, Colegio de Post-graduados de Chapingo, etc.) y destacados botánicos al frente de los mismos (F. Miranda, J. Rzedowski, E. Hernández X., etc.). La investigación realizada era fundamentalmente de índole florística y vegetacional. La primera va ligada al trabajo taxonómico y la segunda al trabajo ecológico.

Los estudios florístico-taxonómicos se incluyen principalmente en las secciones de botánica fanerogámica y criptogámica, aunque durante el 2o. Congreso se crea una sección indefinida y titubeante de florística que se conserva así hasta que, la desaparición de las dos primeras secciones y su asociación con taxonomía le da consistencia y definición.

El $80.7 \%$ del total de trabajos se distribuye entre las siguientes instituciones (Fig. 5 y 6): Instituto de Biología (UNAM)-35\%; Escuela Nacional de Ciencias Biológicas (IPN)-24.8\%; Facultad de Ciencias Biológicas (UANL)-10.5\%; Colegio de Post-graduados de Chapingo-5.2\% e Instituto Nacional de Investigaciones Forestales ( $\mathrm{SAG}$ ) $-5.2 \%$. El resto de los trabajos se produjeron en 20 instituciones dando fe de la gran cantidad de grupos activos así como de su pequeña contribución ( $0.9 \%$ en promedio).

Los estudios vegetacionales-ecológicos se ubican en la sección de ecología que se crea desde el ler. Congreso y en ella se presentan estudios de vegetación que incluyen distribución de formaciones y relación con suelo y clima; esto se consolida cada vez más aumentando su importancia drásticamente durante el 5o. Congreso con el surgimiento de investigaciones en ecología experimental en áreas de ecofisiología, ecología química, interacción planta-animal, sucesión secundaria, etc. En este momento se nota también la irrupción de otra área que es ecología de poblaciones la cual aumenta cuantitativamente y se diversifica en los tres últimos congresos teniendo una gran representación en la sección de ecología. El 71.6\% de los estudios de vegetación-ecología se distribuyen así: 
धा Historia, Bibliografía y Colecciones

Enseñanza de la Botánica

Ecología

Fisiología y Fitoquímica

Taxonomía y Florística

Anatomía y Morfología

Genética y Evolución

Paleobotánica

Botánica Económica

Etnobotánica

Fitogeografía

T O T A L E S

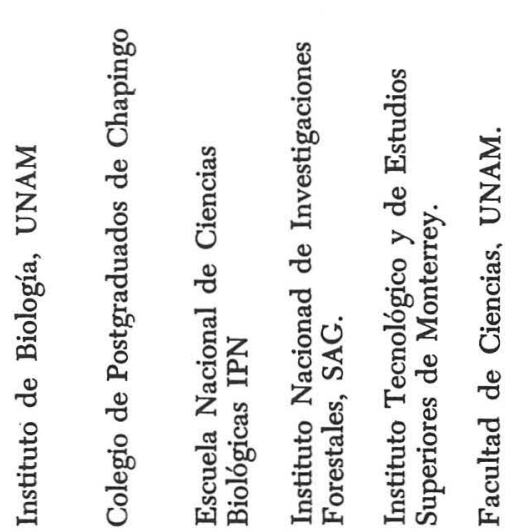

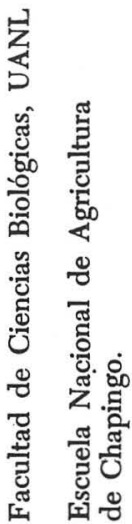
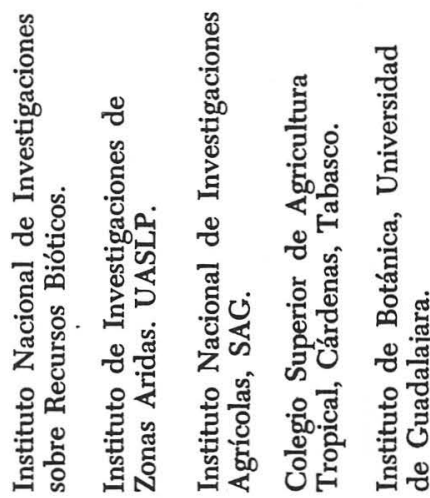

옹

$\begin{array}{rrrrrrrrrrrrrr}11 & - & 1 & 1 & 1 & 2 & - & - & - & 3 & - & - & - \\ 2 & 3 & 1 & - & - & 3 & - & 2 & - & - & - & - & - \\ 78 & 13 & 11 & 28 & 3 & 16 & 6 & 9 & 13 & 5 & - & 6 & 6 \\ 9 & 12 & 7 & 1 & 14 & - & 5 & 9 & 2 & - & 1 & - & - \\ 47 & 7 & 33 & 7 & 5 & 2 & 14 & 1 & 4 & 3 & 2 & 1 & 3 \\ 13 & 13 & 4 & 13 & - & 3 & 2 & 2 & 1 & 1 & - & - & 1 \\ 3 & 18 & 2 & - & 1 & - & - & - & - & - & 6 & - & 1 \\ - & - & 3 & - & - & 1 & - & 1 & - & - & - & - & - \\ 6 & 16 & 3 & 10 & 10 & 1 & 4 & - & - & 3 & 4 & - & 1 \\ 7 & 5 & 1 & 1 & 1 & 7 & 3 & - & 3 & - & 1 & 6 & - \\ 1 & - & 3 & 4 & 1 & - & 1 & - & - & - & - & - & - \\ 177 & 87 & 69 & 65 & 36 & 35 & 35 & 24 & 23 & 15 & 14 & 13 & 12\end{array}$

Fig. 5. Instituciones con mayor participación en los siete congresos (se incluyen las instituciones de los coautores). 

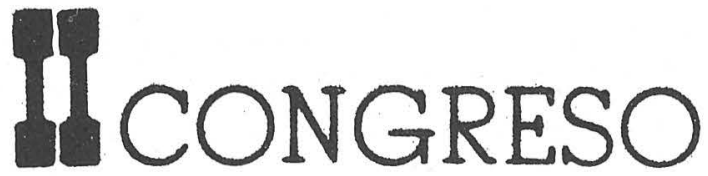

MEXICANO DE BOTANICA

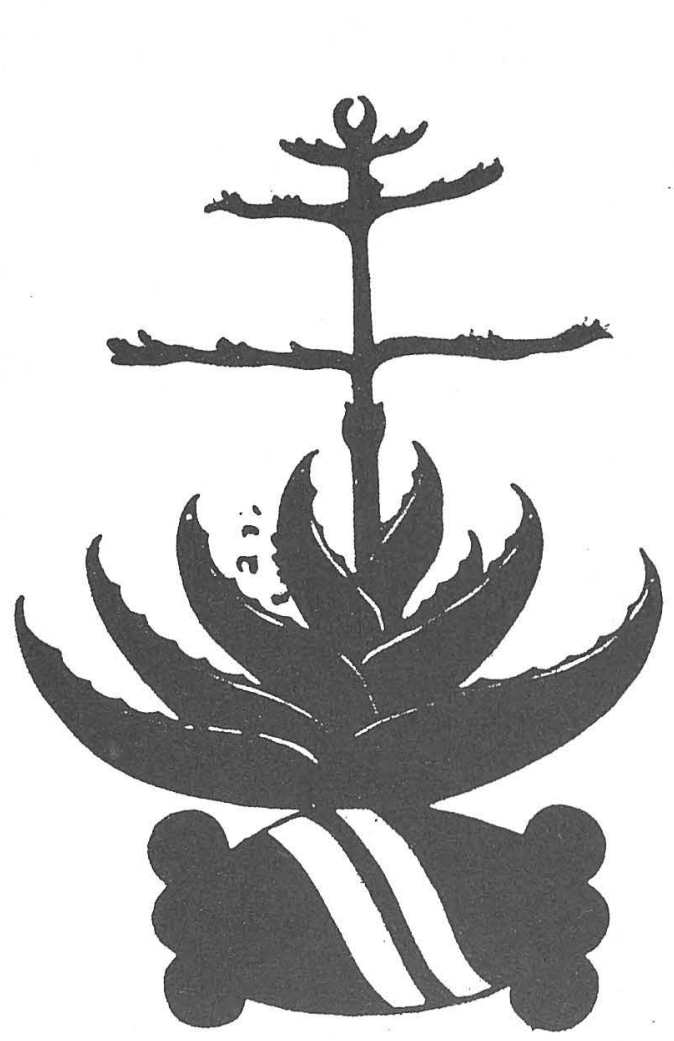

SAN LUS POTOSI

17. al 21

E SEPTEMBRE

03

$\therefore$ : ried Botánica

:... Mexico

Antituto de

investigación

de Zonas Desertici

Universidad

Autónoma do

San Luis Potost

Cartel del Segundo Congreso 
Instituto de Biología (UNAM) - 37.8\%; Instituto Nacional de Investigaciones Forestales (SAG)-13.5\%; Facultad de Ciencias (UNAM) -7.7\%; Colegio de Postgraduados Chapingo-6.3\% e Instituto Nacional de Investigaciones sobre Recursos Bióticos 6.3\%. El resto de los trabajos se produjeron en 23 instituciones manifestando nuevamente la gran dispersión de los grupos y su bajo rendimiento (1.2\% por institución).

La sección de Historia, Bibliografía y Colecciones se ha nutrido del trabajo generado en la consolidación de los herbarios y sistematización de la información preliminar a los estudios florísticos y ha aumentado gradualmente desde el ler. Congreso al último. El $66.5 \%$ se realiza en: Instituto de Biología (UNAM) $-45.8 \%$ Instituto de Investigaciones de Zonas Aridas (UASLP) $-12.5 \%$ y Facultad de Ciencias (UNAM)-8.2\%. Esto refleja en el primer caso, la presencia del Herbario Nacional (MEXU) y la gran actividad en el área florística taxonómica, 35\% de la producción total. El resto de los trabajos se realizó en 9 instituciones y siempre ligados al desarrollo de herbarios, colecciones o estudios florísticos.

Grupo Botánica Económica-Etnobotánica:En la sección de Botánica Económica creada desde el primer congreso se han agrupado trabajos agrícolas, agropecuarios, forestales, industriales, farmacológicos y etnobotánicos. No se observa un incremento del número de trabajos presentados en cada evento lo cual se puede deber a la atomización de la sección en varias otras secciones y/o del criterio utilizado para distribuir los trabajos en las distintas secciones de cada Congreso. Es más, se puede observar un incremento del 1o. al 20. Congreso, después existe una disminución sensible que termina en el 5o. Congreso con la separación de la sección Etnobotánica. Desde entonces aparece sólo esta última mostrando uń incremento notable del número de trabajos presentados. Esta situación es el claro reflejo del surgimiento y desarrollo de grupos de investigación de diversas instituciones (UNAM, Chapingo, IMEPLAN, IPN, etc.). Los trabajos presentados se distribuyen así: En Botánica Económica el 61.7\% del total lo producen: Colegio de Postgraduados de Chapingo-23.5\%; el Instituto Tecnológico de Monterrey-14.7\%; Instituto Nacional de Investigaciones Forèstales (SAG) $-14.7 \%$; Instituto de Biología (UNAM) - 8.8\%; el resto de los trabajos se producen en 15 instituciones con un promedio de $2.5 \%$ cada una. En cuanto a Etnobotánica el $52.2 \%$ se distribuye: Instituto de Biología (UNAM)-15.2\%; Facultad de Ciencias (UNAM)-15.2\%; Colegio Superior de Agricultura de Cárdenas, Tabasco -13\%; Colegio de Postgraduados de Chapingo-10.8\%. El resto se produce en 13 instituciones promediando $3.6 \%$ cada una.

Grupo Anatomía y Morfología: Esta sección se creó desde el primer congreso y se nota un aumento del número de trabajos de manera más o menos regular; es una de las secciones más consistentes. El 64.8\% del total se produce en: Instituto de Biología (UNAM) 21.6\%; Colegio de postgraduados, Chapingo-21.6\% e Instituto Nacional de Investigaciones Forestales (SAC) $-21.6 \%$. El resto se produce en 12 instituciones con un promedio de $2.9 \%$ por cada una.

Crrupo Paleobotánica-Palinología: La sección se inicia vigorosamente durante el ler. Congreso desapareciendo intermitentemente y disminuyendo su número de trabajos, notándose hacia los últimos congresos la presencia de numerosos trabajos de palinología. La actividad en general, en esta área ha estado muy restringida. 


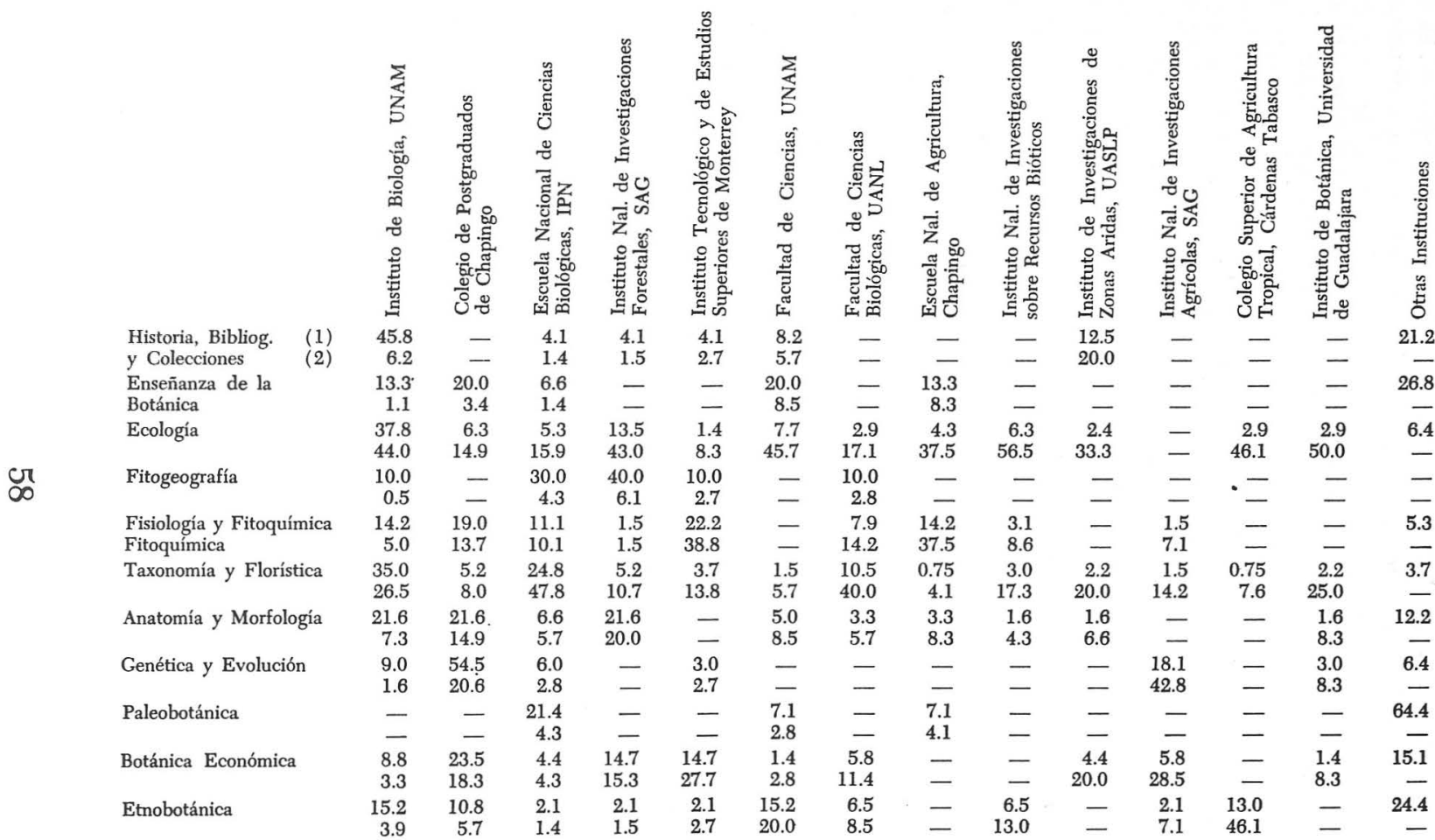

Fig. 6. Participación de las instituciones en las diferentes áreas:

1. Porcentaje de participación o trabajos por área por cada institución.

2. Participación de la institución en cada área con respecto al total de trabajos que ha presentado. 
El 70.6\% se produce en: Instituto de Geología (UNAM)-35\%; Escuela Nacional de Ciencias Biológicas (IPN)-21.4\%; Facultad de Ciencias (UNAM)-7.1\% y Escuela Nacional de Agricultura de Chapingo-7.1\%. El restante se produce en tres instituciones.

Grupo Fisiología-Fitoquímica: La sección de Fisiología se crea durante el ler. Congreso y se mantiene disminuyendo un poco el número de trabajos durante el 2o. y 3o. Se incrementa considerablemente a partir del 4o. cuando se reúne con Fitoquímica progresando hasta el 6o. Congreso cuando se separan en secciones diferentes. El 80.7\% de la producción se distribuye: Instituto Tecnológico de Monterrey-22.2\%; Colegio de Postgraduados Chapingo-19\%; Escuela Nacional de Agricultura de Chapingo-14.2\% y Escuela Nacional de Ciencias Biológicas (IPN) -11.1\%. El 19.3\% restantes se distribuye entre 10 instituciones. Es claro que la mayor parte de estos trabajos está ligado a las instituciones de investigaciones agrícolas.

Grupo Genética y Evolución: Esta sección aparece desde el ler. Congreso aumentado nontablemente hacia el 3o. y después disminuye nuevamente. $\mathrm{Ha}$ incluido trabajos acerca de fitomejoramiento y determinación de números cromosómicos y ha estado ligado fundamentalmente a instituciones de investigación agropecuaria. Durante el último congreso desapareció como sección por la baja participación de este tipo de instituciones. El 81.6\% de la producción se distribuye en: Colegio de Postgraduados de Chapingo-54.5\%; Instituto Nacional de Investigaciones Agrícolas (SAG)-18.1\% e Instituto de Biología (UNAM)-9\%. El restante $18.4 \%$ se reparte entre 6 instituciones promediando $3.1 \%$ cada una.

Grupo Enseñanza de la Biología: Se crea durante el 1er. Congreso y desaparece resurgiendo en el 5o. y aumentando notablemente hacia el 7o. Congreso. A pesar de que el análisis de la enseñanza de la botánica ha sido objetivo de varios congresos, el número total de participaciones ha sido muy bajo y disperso cubriendo desde actividades de difusión hasta técnicas de enseñanza. Las instituciones que más han contribuido son: Colegio de Postgraduados de Chapingo-20\%; Facultad de Ciencias (UNAM)-20\%; Instituto de Biología (UNAM) 13.3\% y Escuela Nacfonal de Agricultura de Chapingo-13.3\%. El resto (33.4\%) lo producen 5 instituciones.

Simposios, mesas redondas y conferencias: Estos han respondido a inquietudes e intereses de los botánicos en general y se pueden agrupar de la siguiente manera:

1. Problemas básicos para el desarrollo del trabajo teórico y práctico en temas tales como: nomenclatura y clasificación de tipos de vegetación, vegetación de México, zonas áridas de México (Congresos I, II y III).

2. Relación entre el trabajo botánico y la realidad nacional: aportaciones a los programas de desarrollo, evaluación de la investigación realizada, utilización y conservación de recursos naturales (Congresos IV, V y VI).

3. Avances en ciertas áreas de la botánica: paleobotánica, florística, sistemática y evolución, fisiología y autoecología, sinecología, dinámica de poblaciones, polinización y etnobotánica (Congresos V y VII).

4. Enseñanza de la botánica (Congresos I, V. VI y VII).

A continuación analizaremos la participación que han tenido las instituciones 
en cada uno de los temas, el número de trabajos por autor y su ubicación por tema e institución. Esto con el fin de entender el momento en que se encuentra cada una de las disciplinas incluidas en el congreso así como su desarrollo inmediato.

Participación de las instituciones: Hemos separado en dos grupos a las instituriones que han participado en función del número de trabajos presentados; así tenemos instituciones con más de 12 trabajos (Fig. 5 y 6) e instituciones con menos de 12 (Fig. 7).

Nuestra discusión la centraremos en el conjunto de instituciones más productivas, 13 de las cuales producen el $83 \%$ del trabajo total, mientras que el conjunto menos productivo incluye 41 instituciones que producen el $17 \%$ del total. Las instituciones más productivas globalmente lo son también por sección; "esto significa que poseen en conjunto el mayor porcentaje de trabajo por sección. Con excepción de palebotánica, donde el Instituto de Geología (UNAM) posee el 35\% del total.

Agrupando los temas por el número de instituciones que han participado podemos hacer tres secciones:

1 a 5 instituciones

6 a 10 instituciones

11 a 13 instituciones
Paleobotánica

Historia, bibliografía y colecciones

Enseñanza de la Botánica

Fisiología y Fitoquímica

Genética y Evolución

Ecología

Taxonomía y Florística

Botánica Económica

Anatomía y Morfología

Etnobotánica

El número de instituciones que participan en cada tema es un indicador de la actividad en cada área lo cual se traduce en el número de grupos y/o individuos que realizan la investigación. La mayor actividad se realiza en ecología, taxonomía y florística. Esto corresponde a la proliferación y diversificanción de los grupos originales establecidos en la UNAM e IPN. En este grupo queda considerada también botánica económica, incluida por la relativamente alta proporción de instituciones de investigación agrícola y forestal, así como por una cierta actividad en este tema que se ha desarrollado esporádicamente en algunas otras instituciones no especializadas. La etnobotánica, al principio ubicada en unas cuantas instituciones, recientemente se realiza en un gran número de ellas. En cuanto a la anatomía y morfología parecería que ha colaborado o alternado con temas florísticos y taxonómicos desarrollándose paralelamente en las instituciones más destacados en esos temas. En el segundo grupo tenemos áreas donde ha habido una participación errática o un reciente abandono del congreso como fisiología, fitoquímica, genética 
Fig. 7. Instituciones que han participado con menos de doce trabajos.

Asociación Mexicana de Orquideología $\ldots \ldots \ldots \ldots \ldots \ldots \ldots \ldots \ldots \ldots \ldots$

Centro de Ecodesarrollo (CONACYT) .................... 1

Centro Internacional de Mejoramiento de Maíz y Trigo ............. 4

Centro de Investigación en Química Aplicada (Saltillo, Coah.) . . . . . . . 1

Centro Regional de Germoplasma Forestal (Chis.) ............... 1

Comisión Forestal del Estado de Michoacán .................. 4

Comisión Nacional de Energía Nuclear .................... 4

Dirección de Nuevos Centros de Población Ejidal, S.R.H. ............ 1

Escuela de Agricultura de Chihuahua. ..................... 1

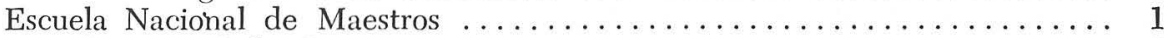

Escuela Normal de Guanajuato $\ldots \ldots \ldots \ldots \ldots \ldots \ldots \ldots \ldots \ldots \ldots \ldots \ldots \ldots$

Individuos (sin representación Inctitunional) $\ldots \ldots \ldots \ldots \ldots \ldots \ldots \ldots$

Instituto de Ecología, A.C. ......................... 2

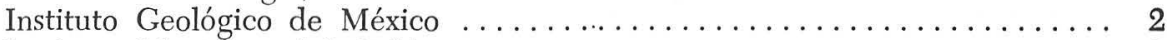

Instituto Mexicano del Café ......................... 2

Instituto Mexicano de Estudio de Plantas Medicinales . . . . . . . . . . . . 3

Instituto Mexicano de Recursos Naturales Renovables ............... 1

Instituto Nacional de Antropología e Historia $\ldots \ldots \ldots \ldots \ldots \ldots \ldots \ldots \ldots . \ldots \ldots$

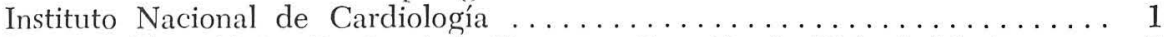

Instituto Tecnológico Regional de Veracruz. Estación de Biología Marina . . . 1

Secretaría de Agricultura y Ganadería. Sanidad Vegetal ............... 1

Secretaría de Educación Pública. Dirección de Culturas Populares ........ 4

Secretaría de Industria y Comercio. Dirección General de Pesca . . . . . . . . . 1

Secretaría de Programación y Presupuesto. Dirección de Estudios del Territorio

Nacional

Secretaría de Recursos Hidráulicos ............................ 1

Sociedad Botánica del Estado de Jalisco .................... 2

Sociedad de Ciencias Naturales de Jalisco ..................... 2

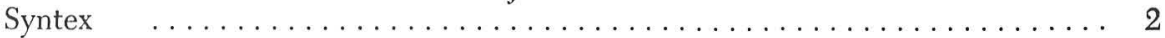

Petróleos Mexicanos ................................ 2

Universidad Autónoma Agraria "Antonio Narro" ................ 7

Universidad Autónoma de Aguascalientes. Centro Agropecuario ......... 1

Universidad Autónoma del Estado de Morelos. Facultad de Ciencias Biológicas 3

Universidad Autónoma de Guerrero. Facultad de Ciencias Químicas y Biológicas 2

Universidad Autónoma Metropolitana

Plantel Iztapalapa $\ldots \ldots \ldots \ldots \ldots \ldots \ldots \ldots \ldots \ldots \ldots \ldots \ldots \ldots$

Plantel Xochimilco .......................... 4

Universidad Autónoma de Nuevo León.

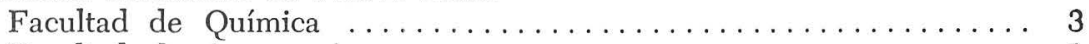

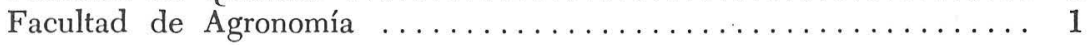

Universidad Autónoma de San Luis Potosí

Escuela de Biología $\ldots \ldots \ldots \ldots \ldots \ldots \ldots \ldots \ldots \ldots \ldots$ 
BOLETIN DE LA SOCIEDAD BOTANICA DE MEXICO No. 40, 1981

Universidad de Guadalajara

Facultad de Agronomía $\ldots \ldots \ldots \ldots \ldots \ldots \ldots \ldots \ldots \ldots \ldots \ldots$

Universidad Michoacana de San Nicolás de Hidalgo

Escuela de Biología ....................... 2

Universidad Nacional Autónoma de México

Colegio de Ciencias y Humanidades Plantel Sur ............. 1

Escuela Nacional de Estudios Profesionales Iztacala . . . . . . . . . 1

Escuela Nacional de Estudios Profesionales Zaragoza ............. 2

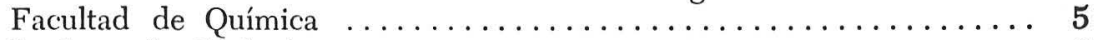

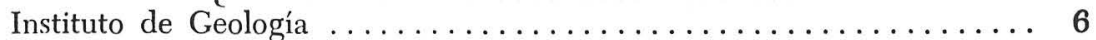

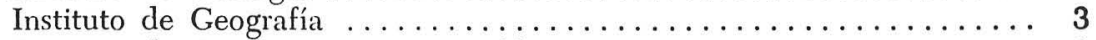

Instituto de Investigaciones Biomédicas $\ldots \ldots \ldots \ldots \ldots \ldots \ldots \ldots, 3$

Universidad de Sonora

Centro de Investigaciones Científicas y Tecnológicas $\ldots \ldots \ldots \ldots \ldots$. 3

Universidad Veracruzana

Escuela de Ciencias Biológicas $\ldots \ldots \ldots \ldots \ldots \ldots \ldots \ldots \ldots \ldots \ldots$ 
y evolución; así como áreas donde la actividad está ligada a intereses más particulares de las instituciones como planes y programas de enseñanza y expansión y consolidación de colecciones biológicas, como es el caso de la enseñanza de la botánica e historia, bibliografía y colecciones respectivamente. Por último el tercer grupo incluye temas donde existe muy poca actividad: paleobotánica y fitogeografía.

Ahora considerando los temas que ocupan el primero y segundo lugar en la producción total de cada institución tenemos:

$\begin{array}{lr}\text { Ecología } & 10 \text { instituciones } \\ \text { Taxonomía y Florística } & 6 \text { instituciones } \\ \text { Botánica Económica } & 5 \text { instituciones } \\ \text { Fisiología y Fitoquímica } & 2 \text { instituciones } \\ \text { Genética y Evolución } & 2 \text { instituciones } \\ \text { Etnobotánica } & 2 \text { instituciones } \\ \text { Historia, Bibliografía y Colecciones } & 1 \text { institución }\end{array}$

Enseñanza de la botánica, fitogeografía, anatomía, morfología y paleobotánica no forman parte de la actividad principal en ninguna institución lo cual es reflejo de su estancamiento o de su franca retracción.

Esto confirma esencialmente lo anotado en las consideraciones anteriores, esto es: ecología, taxonomía y florística son los temas con mayor actividad tanto en centros de investigación y enseñanza básica como en centros de trabajo agrícolaforestal. La botánica económica se restringe como actividad principal en los centros que desarrollan este tipo de investigación. Fisiología, fitoquímica, genética y evolución se realizan principalmente en dos instituciones cada una y etnobotánica, que forma parte del grupo más activo por el número de instituciones donde se lleva a cabo, refleja su juventud con solo dos instituciones.

A continuación enumeramos las instituciones que poseen los registros más altos por sección o tema indicando cuales son éstas (Fig. 4).

El Instituto de Biología (UNAM) : historia, bibliografía y colecciones; ecología, taxonomía y florística; anatomía y morfología y etnobotánica.

Colegio de Postgraduados de Chapingo: enseñanza de la botánica; genética y evolución y botánica económica.

Instituto Nacional de Investigaciones Forestales: fitogeografía y anatomía y morfología.

Facultad de Ciencias (UNAM) : enseñanza de la botánica y etnobotánica.

Instituto Tecnológico de Monterrey: fisiología y fitoquímica.

Cabe hacer ciertas aclaraciones sobre estos últimos datos:

a) La Escuela Nacional de Ciencias Biológicas, IPN, posee altos porcentajes sobre todo en taxonomía, florística, paleobotánica y fitogeografía, sin embargo quedan un poco por debajo de los registros más altos, lo cual no impide que ocupe la tercera posición en número total de trabajos. (Fig. 3).

b) En fitogeografía, enseñanza de la botánica y paleobotánica el número de presentaciones ha sido pequeño $(10,14$ y 15 respectivamente) por lo 
BOLETIN DE LA SOCIEDAD BOTANICA DE MEXICO No. 40, 1981

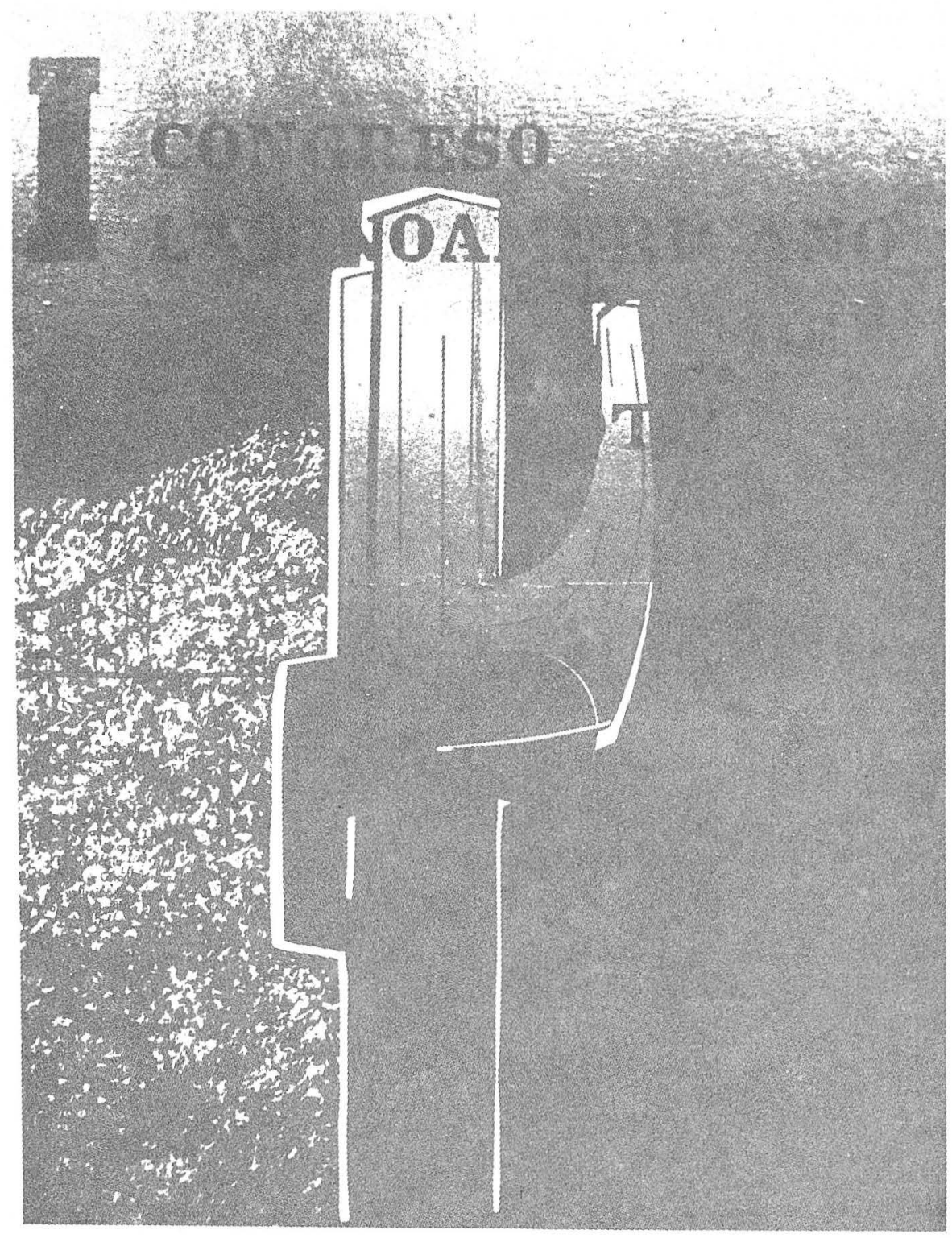

\section{Cartel del Cuarto Congreso}


tanto un solo trabajo hace una diferencia importante en los porcentajes, que no se debe considerar significativo.

Participación por autor. Con el fin de cuantificar la consistencia de los autores que participen en cada área o tema hemos analizado el número de trabajos presentados por cada uno y el tema en el que se incluyen. Suponemos que los más consistentes (fidelidad a un tema y alto número de trabajos) corresponden a los grupos.más sólidos, mejor estructurados y con más recursos. La distribución es la siguiente:

No. de publicaciones

1
2
3
4
5
$6-10$
$11-15$
16
24
34
37

No. de personas

444
66
30
13
5
23
9
1
1
1
1

Como se puede observar existe una enorme cantidad de autores que han presentado un solo trabajo. Esto puede indicar que:

a) Hay una enorme participación (74.7\%) de jóvenes botánicos que llevan al congreso un solo trabajo que puede ser de tesis.

b) La brusca disminución del número de autores con más de un trabajo indica la reciente incorporación de botánicos a las actividades de investigación y la poca edad o consolidación de los grupos de donde forman parte.

c) La cantidad de autores (4) que han publicado más de dieciséis trabajos significa que en el panorama botánico existen sólo unas cuantas personas con grupos bien constituidos, recursos y gran capacidad académica.

Ahora, tomando en cuenta como autores más productivos aquellos, con más de 6 trabajos presentados, lo cual equivaldría a un promedio de un poco menos de uno por congreso, tendrenos sólo 36 autores. Distribuyendo su participación por temas tenemos:

$\begin{array}{ll}\text { Florística } & -17 \text { autores } \\ \text { Ecología } & -13 \text { autores } \\ \text { Taxonomía } & -11 \text { autores } \\ \text { Fisiología y Fitoquímica } & -5 \text { autores } \\ \text { Anatomía y Morfología } & -2 \text { autores } \\ \text { Fitogeografía } & -1 \text { autor } \\ \text { Botánica Económica } & -1 \text { autor }\end{array}$


Se cita un total de 50 autores que con relación a los 36 detectados nos da un promedio de 1.4 por autor lo cual significa una gran fidelidad de los autores por tema. Los temas con autores más productivos son: florística, ecología y taxonomía lo cual, aunado al mayor número de instituciones participantes y a la importancia que tienen como temas principales de actividad por institución los perfilan como las áreas más importantes de la botánica tanto cuantitativa como cualitativamente. Fisiología y fitoquímica aparecen como un trabajo bien estructurado pero limitado sólo a unas cuantas instituciones, lo cual se puede interpretar como área de desarrollo limitado. Anatomía y morfología aunque distribuido en gran cantidad de instituciones no es actividad principal de ninguna de ellas pero posee grupos consistentes. Botánica económica se distribuye en varias instituciones como tema principal de investigación sin embargo, sorprende con sólo un autor productivo. Fitogeografía evidentemente es un tema colateral para los investigadores, aún para los más productivos.

Actividad botánica en provincia: En este punto nos ocupamos de la distribución del trabajo botánico en el país con afán de comparar la actividad en provincia y en el área metropolitana de la cuidad de México.

Area metropolitana

No. total de instituciones

No. de instituciones

más productivas

Porcentaje producido

Autores más productivos
34

26 (entre 6 y 37 trabajos)
Interior del país

28

25

10 (entre 6 y 15
trabajos)

Numéricamente no existe una gran diferencia en la distribución de instituciones, sin embargo comparando el porcentaje producido y el número y rendimiento de los autores encontramos un sesgo marcado hacia el área metropolitana. Esto refleja una mayor tradición y cantidad de recursos disponibles lo que ha creado mejores condiciones de trabajo y estructuración de grupos de investigación más eficientes.

Centros de investigación y centros de docencia-investigación: Intentamos comparar la cantidad que se produce en estos dos tipos de instituciones con afán de interpretarlo en función del impacto que la investigación botánica tiene en los planes y programas de enseñanza de la biología. Reconocemos que no existe una separación o aislamiento de los centros de investigación de los de docencia sin embargo, el efecto que los primeros producen en los segundos no es directo $\mathrm{y} / \mathrm{o}$ inmediato. 
Centros de docenciainvestigación

Número total de instituciones

Instituciones más productivas

Porcentaje producido

Autores más productivos
22

7

36.5

12
Centros de investigación

La comparación pone en evidencia un mayor número de centros de investigación que de docencia aunque encontramos un mayor número de instituciones productivas en la segunda categoría. La producción de làs dos terceras partes se realiza en los centros de investigación; esto significa que existe una gran polarización en donde unos cuantos producen la mayor proporción.

Grupos taxonómicos más atendidos: Para completar el análisis sintetizamos los datos correspondientes a la atención prestada a diferentes grupos de vegetales:

$\begin{array}{lr}\text { *Dicotiledoneas } & 53.2 \% \\ \text { * Monocotiledoneas } & 18.5 \% \\ \text { Gimnospermas } & 5.4 \% \\ \text { Hongos } & 9.7 \% \\ \text { Algas } & 8.0 \% \\ \text { Helechos } & 3.5 \% \\ \text { Liquenes y musgos } & 1.0 \%\end{array}$

- leguminosas, cactáceas y compuestas principalmente * gramíneas y palmas principalmente

De la información acumulada podemos concluir que los estudios de vegetación, florística, taxonomía y ecología son los más abundantes e importantes desde el punto de vista de su participación, posibilidades y grupos de investigación que intervienen. Los estudios ecologicos todavía se encuentran fuertemente ligados a los estudios de vegetación. Sin embargo, debemos hacer notar que desde el 50. congreso se evidencía un cambio importante; esto es, aparece una cantidad considerable de estudios de ecología experimental alrededor de diversos aspectos de la sucesión secundaria como son ecofisiología de la germinación, alelopatía, dispersión y polinización, banco de semillas, etc. También ocupan un lugar importante diverso trabajos de ecología de poblaciones vegetales en zonas templadas y tropicales incluyendo estudios de dinámica de poblaciones, interacción planta-animal, productividad, etc. Este desarrollo augura el nacimiento de una escuela de ecología nacional que genere investigación e ideas originales. Esta situación se presenta sólo en esta área y probablemente en etnobotánica donde 


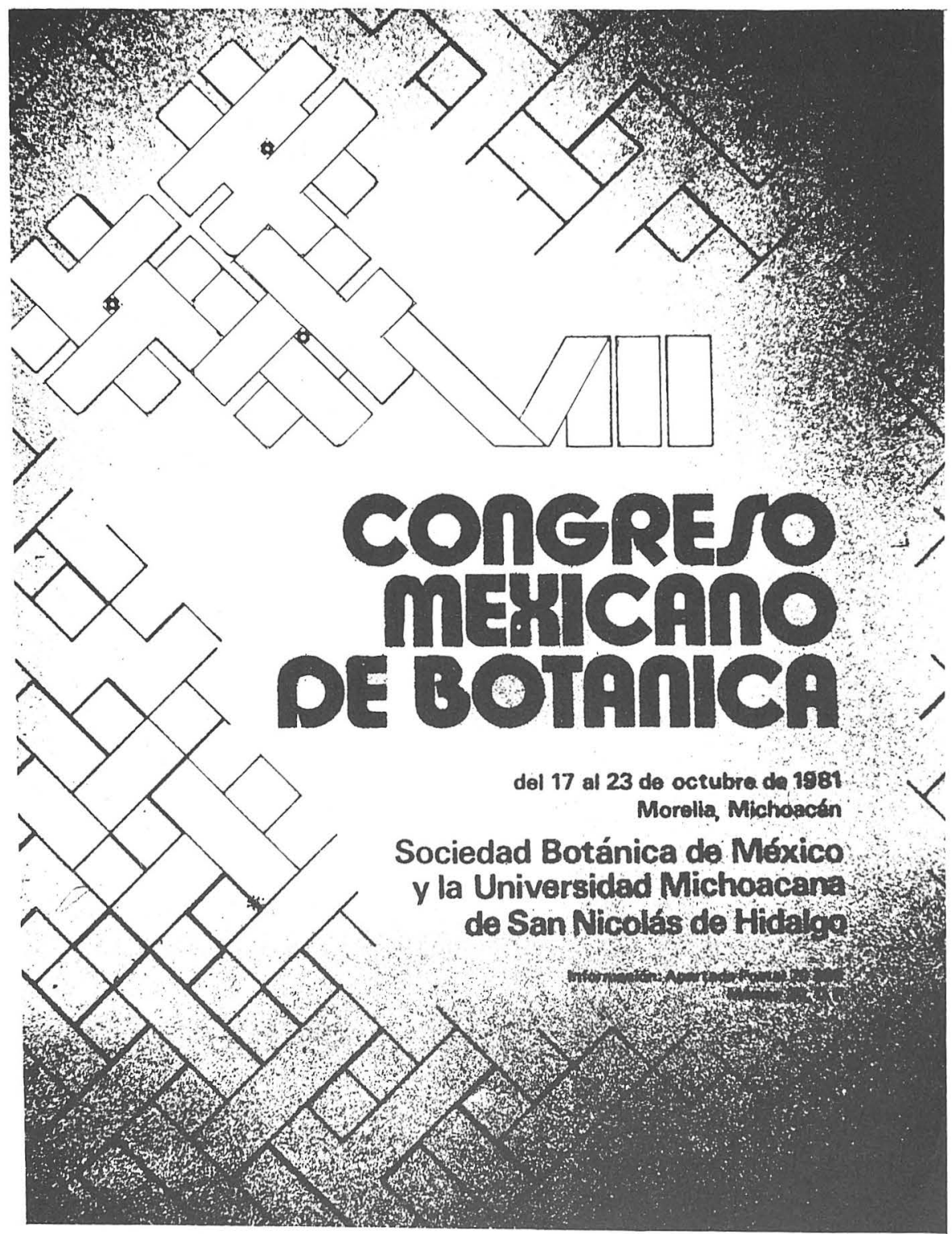

Cartel del Octavo Congreso 
podemos percibir una gran diversificación de grupos en distintas instituciones, así como la presencia de investigadores productivos. Estos grupos han mostrado un gran interés en el análisis conceptual y metodológico, lo cual ha creado un ambiente altamente crítico respecto de la investigación que se realiza. Lo cual podría redundar también en la formación de una escuela nacional.

Los estudios florísticos se realizan en gran número, reflejando el estado en que se encuentra el conocimiento de nuestros recursos vegetales. La taxonomía como tal se ha separado reciéntemente de los estudios florísticos, sin embargo aún se restringe a un pequeño número de grupos y por ende de instituciones. Consideramos que en este momento representa una gran lmiitación al desarrollo de la florística lo cual alcanzará muy pronto su máximo desarrollo permisible bajo esta condición.

La botánica económica ha disminuido su participación recientemente debido fundamentalmente a la promoción que se ha realizado. También debemos aclarar: que es un área poco definida en relación con otras que hemos venido manejando en este trabajo, pues incluye una gran cantidad de participaciones que podrían incluirse en otros apartados disminuyendo o aumentando su representatividad como área. Podemos sin embargo, subrayar que en los estudios agrícolas ha existido una marcada tendencia hacia el filtomejoramiento y en menor escala hacia la fisiología, fitoquímica, etnobotánica y ecología.

Existe un escaso desarrollo de fitogeografía debido probablemente entre otras causas a la situación de la florística y taxonomía y su relación mutua como se mencionó antes, así como al pobre desarrollo de la paleobotánica.

Para terminar señalaremos algunos aspectos que nos parecen importantes:

1. Durante los congresos se han presentado y discutido distintos asuntos o temas de interés y relevancia nacional bajo diversos aspectos como son: inventario y uso de recursos, análisis de investigación y docencia, etc. Sin embargo esto, no ha generado un trabajo continuo al respecto instrumentable a través de comisiones o grupos que enriquezcan la discusión, aporten información o propongan lineamientos. Esto debería ser una preocupación de la Sociedad Botánica de México. Se debe procurar que el Congreso Mexicano de Botánica se convierta en un foro de discusión, análisis y jerarquización de problemas o asuntos de interés general que genere grupos de trabajo que den continuidad a esta labor.

2. El contenido de los congresos ha incluido prácticamente todas las áreas de la botánica en el país. Esto ha promovido un intercambio de información muy útil y benéfico a la investigación que si bien no ha impulsado un fuerte desarrollo interdiciplinario si ha redundado al menos en promover la cooperación entre grupos e instituciones y en mantener en comunicación a los investigadores de diversas disciplinas en distintas zonas del país. Es por esto que se deberá procurar la más amplia participación con el fin de mantener esta situación y fomentar el desarrollo de trabajo interdiciplinario.

3. La enseñanza de la botánica ha sido una inquietud permanente, sin embargo no se ha realizado un esfuerzo encaminado al análisis de la situación 
a nivel del país y de su relación con la investigación con el fin de proponer lineamientos de un plan o programa nacional de formación de recursos humanos.

4. La mayor parte de la investigación botánica se realiza en el área metropolitana de la ciudad de México, situación que debería modificarse impulsando la realización de convenios de participación e intercambio de personal académico en beneficio de las instituciones de provincia y de la eficiencia de programas de investigación regional.

5. Se puede notar que la mayor parte de la investigación se realiza en universidades e institutos y una parte muy reducida en instituciones estatales y paraestatales. Esto se puede explicar por la poca vinculación de la investigación en botánica con los planes nacionales de desarrollo. Existen algunos ejemplos notables como fue la gran actividad de investigación en varias disciplinas que desarrolló el Instituto Nacional de Investigaciones Forestales de la SAG hace algunos años en los trópicos húmedos, programa que dejó de funcionar, desintegrándose su plantel de investigadores y deteriorándose su infraestructura.

Para finalizar queremos transcribir unas líneas de Gómez-Poyou (1975) las cuales se identifican con nuestras ideas en este sentido, "dado que la planeación y desarrollo de la ciencia y la investigación es una responsabilidad que debe asumir la totalidad de la comunidad científica, esto se podría llevar a cabo por medio de las sociedades científicas del país".

\section{RESUMEN}

Se realizó un análisis del contenido de los Congresos Mexicanos de Botánica del I al VII.

Se relacionó la cantidad de trabajos por sesión y el número de autores con el desarrollo de las áreas de investigaciones correspondientes.

Se comparó la actividad bọtánica en centros de docencia e investgiación y en instituciones del centro e interior del país.

Se anotan recomendaciones para el futuro desarrollo del congreso y sus implicaciones en la comunidad botánica del país.

\section{ABSTRACT}

The content of the Mexican Congresses of Botany (I-VII) was analyzed. The number of author taking part and the quantity of papers in each session was related with the development of the corresponding research areas. The botanical activity in research and educational center was compared; also between institutions in the metropolitan area and the interior of Mexico.

Some recommendations for the future development of the Congress and its implications on the mexican botanical community are commented. 


\section{AGRADECIMIENTOS}

Los autores desean agradecer la lectura y comentarios hechos al manucrito por el Dr. Arturo Gómez-Pompa, Ing. Efraím Hern;ndez Xolocotzi, M. en C. Antonio Lot Helgueras, Dr. Jerzy Rzedowski y Dr. José Sarukhan Kermes.

\section{BIBLIOGRAFIA}

GOMEZ-POMPA, A. 1975. La investigación Botánica en México en los últimos diez años. Biología 5 (1-4): 25-30.

GOMEZ-POYOU, A. 1975. La investigación científica y su organización en México, Biología 5 (1-4): 16-20.

HERNANDEZ, X., E. 1961. La biología Agrícola en México. Rev. Soc. Mex. Hist. Nat. XXII: $153-184$.

HERNANDEZ, X., E. 1969a. La Investigación Botánica en el Instituto de Biología de la Universidad Nacional Autónoma de México. Simposio la Investigación Botánica en México. IV Congreso Mexicano de Botánica. MonterreySaltillo. 8-11 de septiembre de 1969.

HERNANDEZ, X., E. 1969b. La Investigación Botánica en la Escuela Nacional de Agricultura y su Colegio de Postgraduados. Simposio La Investigación Botánica en México. IV Congreso Mexicano de Botánica. Monterrey-Saltillo. 8-11 de septiembre de 1969.

MIRANDA, F. 1961. La Botánica en México en el último cuarto de siglo. Rev. Soc. Mex. Hist. Nat. XXII: 85-111.

RZEDOWSKI, J. 1975. Ensayo de Evaluación de la Investigación Botánica en México. Conferencia. IV Congreso Mexicano de Botánica.

SARUKHAN, J. 1975. Los grupos de investigación como unidades de desarrollo científico. Biología 5 (1-4): 3-10.

I Congreso Mexicano de Botánica. Programa y Resúmenes. Octubre, 1960. Memorias, Boletín de la Sociedad Botánica de México No. 29. Agosto, 1963.

II Congreso Mexicano de Botánica. Programa y Resúmenes. Septiembre, 1963.

III Congreso Mexicano de Botánica. Programa y Resúmenes. Octubre, 1966.

IV Congreso Mexicano de Botánica. Programa y Resúmenes. Septiembre, 1969.

V Congreso Mexicano de Botánica. Programa, Resúmenes y Memorias. Diciembre, 1972.

VI Congreso Mexicano de Botánica. Programa y Resúmenes. Septiembre, 1975. VII Congreso Mexicano de Botánica. Programa y Resúmenes. Octubre, 1978. 